\title{
A hybrid objective function with empirical stability aware to improve RPL for IoT applications
}

\author{
Abdelhadi Eloudrhiri Hassani, Aicha Sahel, Abdelmajid Badri, El Mourabit Ilham \\ EEA and TI laboratory, Faculty of Sciences and Techniques, Hassan II University, Casablanca, Morocco
}

\section{Article Info}

Article history:

Received Aug 16, 2020

Revised Sep 6, 2020

Accepted Okt 1, 2020

\section{Keywords:}

Combined metrics

Contiki OS

IoT

Objective function

RPL

WSN

ABSTRACT

The diverse applications of the internet of things (IoT) require adaptable routing protocol able to cope with several constraints. Thus, RPL protocol was designed to meet the needs for IoT networks categorized as low power and lossy networks (LLN). RPL uses an objective function based on specific metrics for preferred parents selection through these packets are sent to root. The single routing metric issue generally doesn't satisfy all routing performance requirements, whereas some are improved others are degraded. In that purpose, we propose a hybrid objective function with empirical stability aware (HOFESA), implemented in the network layer of the embedded operating system CONTIKI, which combines linearly three weighty metrics namely hop count, RSSI and node energy consumption. Also, To remedy to frequent preferred parents changes problems caused by taking into account more than one metric, our proposal relies on static and empirical thresholds. The designed HOFESA, evaluated under COOJA emulator against Standard-RPL and EC-OF, showed a packet delivery ratio improvement, a decrease in the power consumption, the convergence time and DIO control messages as well as it gives network stability through an adequate churn.
\end{abstract}

This is an open access article under the CC BY-SA license.

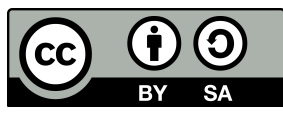

\section{Corresponding Author:}

Abdelhadi Eloudrhiri Hassani

EEA and TI laboratory

Faculty of Sciences and Techniques, Hassan II University, Morocco

Email: eloudrhiri.abdelhadi@gmail.com

\section{INTRODUCTION}

The internet of things (IoT) is a wireless communication technology with a great potential for humanity which provides access facilities to the physical world everywhere all the time. Things term in IoT make direct reference to networked embedded devices with sensors and actuators [1]. These networks embrace a large number of battery-powered devices with constraints in term of processing limitations and storage capacity [2]. Systems based on the Internet of Things have countless applications such as smart cities [3], smart home [4], healthcare [5, 6], industries [7] and smart grids [8]. Thereby, IoT networks are expected to ensure efficiency and reliability in the future even if they are deployed in a harsh environments. These networked devices must be apt to handle data processing, packet transmission, and energy consumption according to their limited capacities. Thus, The MAC and routing protocols are required to respond to those challenging tasks.

In that purpose, a routing protocol for low-power and lossy networks (RPL) was designed by the internet engineering task force (IETF) [9]. The RPL is a routing protocol destinated for limited resource IoT platforms, based on IPv6 and uses the IEEE802.15.4 at the PHY and MAC layer [10]. With RPL as a proactive routing protocol, the paths are constructed once the network is initialized. The nodes in the network use the RPL in order to set up a tree-like routing topology which is a destination-oriented directed acyclic graph (DODAG), 
based on four principal ICMPv6 messages: DODAG information object (DIO) holds information that enable nodes to know the instance, configuration and select the preferred parent, DODAG information solicitation (DIS) message as a DIO request from neighbours, DODAG advertisement object packets (DAO) used to collect topology informations and DAO-ACK as a response to a DAO message. The aim of this kind of topology is to steer all the data packets to one or more sink nodes. These routing paths are created using a specific objective function (OF). In the core of RPL, two OF's are proposed namely the minimum rank with hysteresis objective function (MRHOF) [11] based on expected transmission counts (ETX) as a routing metric and OF0 [12], which is based on hop count. The paths in MRHOF are based on the link quality metric calculated by broadcasting probe packets between the sender and receiver nodes at time intervals. On the other hand, the routing paths in OF0 are based on node metric that aims to each node in the network to know its position against the sink. However, the two objective functions tend to minimize the cost of their metrics which causes non-optimized routes due to taking into account a single constraint. Considering those drawbacks, we propose a designed hybrid objective function with empirical stability aware (HOFESA), based on a new method of rank processing using linear combination of radio signal strength indicator (RSSI), hop count (HC) and energy consumption (EC) metrics in order to select the optimal preferred parent taking into account several constraints and stability through an static or empirical threshold. The main contributions of this paper are summarized as follows:

- Enhance the RPL by a newly designed objective function HOFESA. Our proposal combines linearly three different metrics, with different weights, chosen to respect the objective function convergence, cope with different constraints

- Consider the stability in routing by two diferrent thresholds

- A simulation under Cooja of HOFESA compared to Standard-RPL and EC-OF in term of Packet Delivery Ratio, Energy consumption, Convergence time, churn and number of DIO control messages

The rest of this paper is organized as follow. In section 2 we present the related works with RPL routing protocol. In section 3 we present the proposed objective function HOFESA. In section 4 we report the experimental results and discussion, finally a conclusion is given in section 5 .

\section{RELATED WORKS}

The RPL protocol has been the subject of several researches aiming to enhance or adapt it to different requirements since only three metrics are implemented in RPL core i.e. expected transmission count, energy consummed and hop count. Authors were interested first on these objective functions in order to study their advantages and limits [13-15]. However, in term of single metrics, Xiao et al. [16] proposed the average Expected Transmission Count of the path towards the sink. This proposal address the problem of single long hops introduced in high densities but can't cope with power consumption of the network. Remaining energy as a node metric for next hop selection has been defined by kamgueu et al. in [17] to choose parents with the most residual energy. This new metric has proven to be effective in terms of extending the network lifetime and distribute energy evenly among nodes, but do not considers the quality of links which leads to choose lossy paths. In order to cope with bottlenecks issue generated by long hops in high densities, San Martin et al. in [18] proposed Sigma-ETX metric based on the standard deviation of ETX in each route. The best path is that with the minimum number of deviations. However, the energy consumption also is not considered by the authors which conducts to fast nodes depletion. The delay issue was considered by Gonizzi et al. in [19] with a novel objective function based on AVGDELAY metric which builds the network arborescence to minimize the routing average delay between senders and root. The proposal showed a significant decrease in terms of latency but can't cope with reliability performances.

The requirements of each application differs which leaves the single routing metric not the ideal implementation to offer the best quality of routing services. In this context, several works have been proposed based on metrics amalgamation. RPL adaptation for smart grid applications are proposed by Nassar et al. in [20] who designed OFQS objective function which combines ETX, delay and power state metrics. It uses the concept of hysteresis in order to ensure path stability selection and reduce the churn. It showed a lifetime, end to end delay and packet delivery ratio improvements as results. In [21], Gao et al. proposed ETEN-RPL a hybrid routing metric that combine ETX and remaining energy additively injected into an objective function. It remediates to the problems of unbalanced energy and bad data reliability, improves the stability and reduces the power consumption in the network. However, the proposal is limited to performance results only in very 
low densities. Mishra et al. proposed lexicographic and additive approachs in [22] to combine ETX, available energy and hop count routing metrics into EHA objective function for rank processing and select an optimized preffered parent. The results showed better performance in terms of energy consumption, network latency and packet delivery ratio compared to MRHOF-ETX and OF0. In [23], Al-Kashoash et al. have been interested to the paths congestion caused by the buffer nodes occupancy. In that purpose, Congestion-Aware Objective Function CA-OF were be proposed which consider ETX metric at low data rate while the buffer occupancy is considered at high data rate. The proposal is limited to packet delivery ratio, power consumption and don't evaluate other performance parameters also don't consider a large scale of densities.

Many researches used the fuzzy logic as an approch for combined metrics. Indeed, in [24], Araujo et al. proposed a new objective function called DQCA-OF that combines three metrics, i.e. ETX, hop count and energy consumed. DQCA-OF provides a packet delivery ratio over 95\%, reduce end to end delay and the number of excepted transmission count. However, the proposal is simulated with a topology of 20 nodes which need other tests for higher densities. Also in [25], Sankar et al. designed FLEA-RPL objective function based on ETX, load and residual energy, it is used for calculating the step parameter for rank assignment. The proposal shows a rise of PDR around $2 \%$ to $5 \%$ and an increase of lifetime around 10\%. In [26], Lamaazi et al. proposed a new objective function based on fuzzy logic system EC-OF that combines tree metrics: ETX, Hop Count and energy consumption. The results showed that the EC-OF keeps the routing protocol RPL efficient and improve its performances in term of PDR, network life time, convergence time, latency and power consumption against MRHOF.

\section{RESEARCH METHOD}

\subsection{Problem statement}

By its default definition, RPL uses a single routing metric such as ETX, energy consumption or hop count for selecting the preferred parents, which leads to show some limits and poorly performs in applications where different constraints must be taken into account. Indeed, RPL based on one metric minimized by the objective function, conduct to select non-optimized or static routing paths which greatly affects the network quality of service performances. Also, its noteworthy that when the density of network increase, the frequent preferred parents change phenomenon increases too which destabilizes the network.

To overcome these issues, we propose a hybrid objective function with empirical stability aware (HOFESA) based on linear combination of several metrics namely RSSI, Energy consumption and Hop count to cope with different constraints while an empirical Threshold is implemented to give more network stability. Accordingly, HOFESA utilizes diverse policies from the DIO amendment to rank computing and parent selection procedures. To have an optimized parent selection with HOFESA, we have equipped the DIO message with a hop count metric as shown in Figure 1, while RSSI and Energy consumption metrics are locally computed by each node. Our proposed objective function selects the best parent based on certain priority interpreted by weights. Thus, the metric with the highest weight has the most influence in the nodes parent's selection. However, the design of a novel objective function using composite routing metrics must respect the monotonicity property defined by (1) to be loop-free directed to sink.

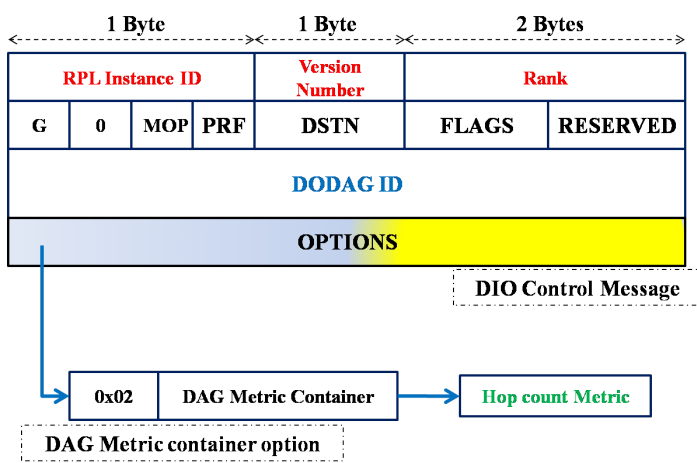

Figure 1. Amended DIO message with hop cout metric 


$$
\left\{\begin{array}{l}
(g 1(\lambda), g 2(\lambda))<(g 1(\Theta), g 2(\Theta)) \Leftrightarrow g 1(\lambda)+g 2(\lambda)<g 1(\Theta)+g 2(\Theta) \\
(g 1(\lambda), g 2(\lambda))>(g 1(\Theta), g 2(\Theta)) \Leftrightarrow g 1(\lambda)+g 2(\lambda)>g 1(\Theta)+g 2(\Theta)
\end{array}\right.
$$

Where $\lambda, \Theta$ are two different routing paths and $\mathrm{g} 1, \mathrm{~g} 2$ are the functions which define the primary routing metrics.

\subsection{Metrics of interest}

In our case, the primary metrics must be chosen in such a way that all must be minimized for HOFESA convergence adn respect (1).

\subsubsection{Hop count metric:}

It is a metric that shows the number of nodes in a routing path, minimizable by the objective function to find the shortest path to sink and processed as in (2).

$$
H o p C o u n t(i)= \begin{cases}\text { MinHC_Increment } & \text { if } c p=\operatorname{sink} \\ H C(c p)+\text { MinHC_Increment } & \text { if } c p \neq \operatorname{sink}\end{cases}
$$

Where $\mathrm{cp}$ is the candidate parent susceptible to be the preferred parent of node $\mathrm{i}$ and the value of MinHC_Increment is 256.

\subsubsection{Energy consumption metric:}

It is a metric that compute the current power consumption by each node. It is must be minimizable by the objective function in order to choose preferred parents with lower energy consumption. It is processed as in (3).

$$
E C(i)=\frac{C P U * 5.4+\text { Trans } * 58.5+\text { List } * 64.5+L P M * 0.1635}{32768}
$$

Where CPU,Trans,List,LPM are respectively the number of ticks when the node is in CPU level processing, transmitting, listening or going to low power mode [27], while numerical parameters are the nominal values provided in the Skymote Datasheet.

\subsubsection{RSSI metric:}

it is CC2420 radio metric based on the signal strength and provided through an RSSI_register adjusted with antenna variation named offset modeled during the system development. The RSSI is computed as (4). It is a maximizable metric in order to give the best link between node and neighbor node since it is measured with a logarithmic scale in $\mathrm{dBm}$, typically ranges between $0 \mathrm{dBm}$ and $-110 \mathrm{dBm}$ respectively for a very strong and low signal levels. It is a metric to maximaze while hop count and energy consumption metrics must be minimized. Thus, to respect the designed objective function monotonicity, we used the inverse of RSSI.

$$
R S S I(i)=R S S I \_r e g i s t e r(i)-45
$$

\subsection{Design of hybrid objective function with empirical stability aware (HOFESA)}

The HOFESA is based on novel method of rank processing. Indeed, at a DIO reception from candidate parent, the node measure the RSSI at the MAC layer following (4), it energy consumption following (3) and add the candidate parent hop count value advertised in the DIO message to the MinHC_Increment as in (2). After all these computations, since the metric priority is interpreted by weights, the increment of rank is calculated as in (5). Finally, the node compute its susceptible new rank refering to (6).

$$
\text { RankIncrement }=H C(i)+\alpha * R S S I(i)+\beta * E C(i)
$$

Where $\alpha$ and $\beta$ are the weights influence of each metric comprised between 0 and 1 , while the hop count have a constant weight equal to 1 . Also, the weights of $\alpha$ and $\beta$ must be complementary such a way that their summation is equal to 1 . 


$$
\operatorname{Rank}(i)=\operatorname{Rank}(c p)+\text { RankIncrement }
$$

Where the Rank(cp) is the rank extracted from the candidate parent DIO.

Once the node had process its susceptible new rank, if it don't already have a preferred parent, the node opt for the new calculated rank. Otherwise, a comparison of the susceptible new rank with the preferred parent rank is inevitable, if it is higher, then the candidate parent is discarded. Else, for stability awareness, if it is lower than the preferred parent rank with a certain threshold, then the candidate parent is retained. The threshold is essentiel for reducing the preferred parent changes. For that purpose, the Static Threshold value defined in (7) is used as proper HOFESA threshold while the Empirical threshold defined in (8) is utilized to optimize the performances of our proposal by giving more stability.

$$
\begin{gathered}
\text { StaticThreshold }=\text { MinHC_Increment }+\frac{\text { MinHC_Increment }}{2} \\
\text { EmpiricalThreshold }=\text { StaticThreshold }+ \text { Evalue }
\end{gathered}
$$

Where the Evalue is defined during the simulations which gives the best performance optimization.

At this stage, if the preferred parent change condition is fulfilled, the node update it metric container and rank in the DIO message and broadcast a new one. Table 1 describes the proposed HOFESA algorithm.

Table 1. Proposed HOFESA algorithm

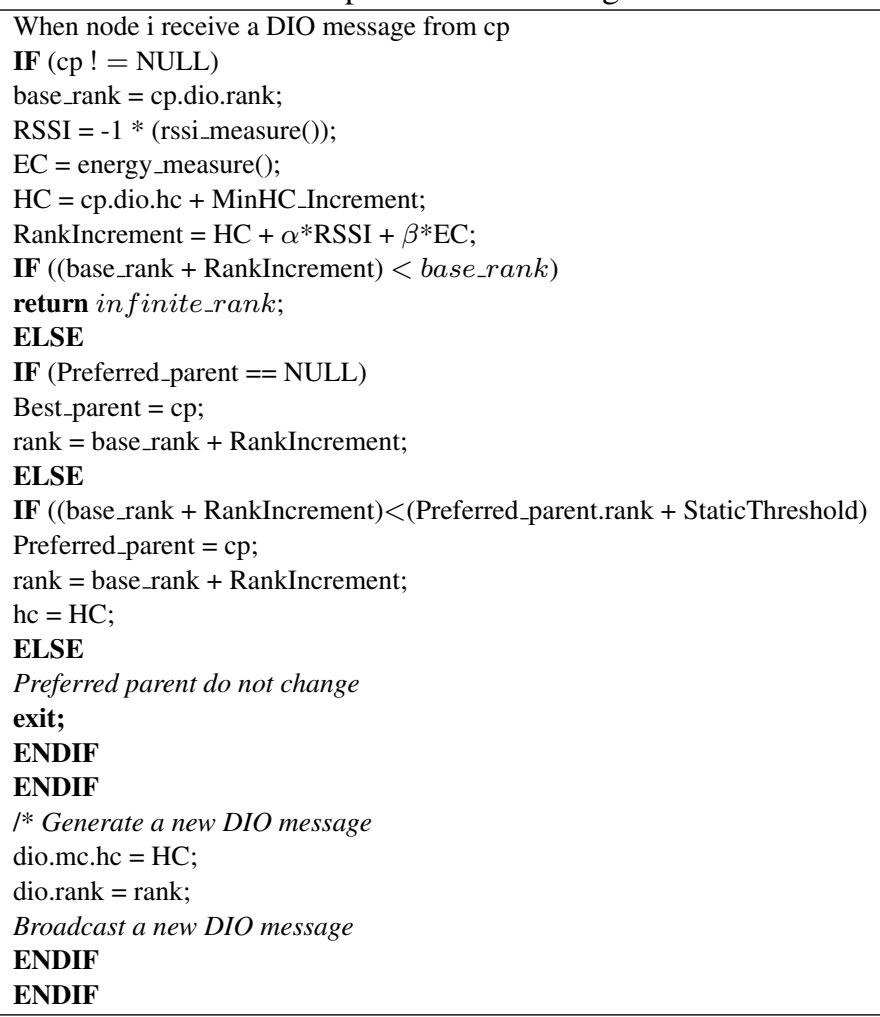

\section{RESULTS AND DISCUSSION}

This section provide a discussion about the outcome obtained from the proposed implementation of hybrid objective function with empirical stability aware. In order to evaluate our proposal, we have exploited the simulation environment COOJA. It is considered as an emulator of networked embedded platforms running 
contiki as an embedded operating system. Our simulations are established with the IoT skymotes platforms that use the low power Texas instruments MSP430 micro-controller as CPU and employs for its wireless communications the radio module chipcon CC2420. In this work, the skymotes are randomly deployed in an area of 200x200 m. Different densities are considered from 25 to 100 senders with a single sink that collects all the data of the network. The efficiency of our proposed method has been investigated through its comparison against standard RPL and EC-OF in terms of five performance metrics namely packet delivery ratio, power consumption, convergence time, churn and number of DIO control packets. Also, in order to give an idea about the impact of $\alpha$ and $\beta$ parameters in the rank calculation by our objective function, we took three different values $0.3,0.5$ and 0.7 as weights. The simulation is performed over $600 \mathrm{~s}$ for every environmental setup. Simulation parameters are summarized in Table 2. However, this section is divided on two parts, the first where we analyse and discuss the performances provided by our proposal in term of network density, while the second we focus more on the HOFESA stability using the empirical threshold.

Table 2. Simulation parameters

\begin{tabular}{lc}
\hline Network simulator & Cooja \\
Embedded operating system & Contiki 2.7 \\
Radio environment & Unit disk graph medium - DL \\
Emulated nodes & Sky motes \\
Network area & $200 \times 200 \mathrm{~m}$ \\
Deployment of nodes & Random \\
Number of senders & $25,50,100$ \\
Evalue & 200 \\
Number of sinks & 1 \\
Transmission / interference ranges & $70 / 100 \mathrm{~m}$ \\
Objective functions & RPL-standard, EC-OF \\
Simulation time & $600 \mathrm{~s}$ \\
\hline
\end{tabular}

\subsection{Performance evaluation of HOFESA}

\subsubsection{Analysis of packet delivery ratio}

The network reliability of HOFESA is assessed by comparing it to Standard RPL and EC-OF in terms of PDR for different densities. As noticed in Figure 2, the EC-OF provide a low PDR due to it tend to choose nodes with lower power consumption despite of poor link quality. Regarding standard-RPL that tends to minimize the ETX metric aiming to offer routing paths with good link quality, it cannot perform better against our proposed method. It can be explained by the fact that the several routing metrics hybridization increases the number of routing paths, thus avoiding the bottlenecks responsible in packets loss. Another aspect that explains this improvement is the number of preferred parent changes indicated by the churn. Indeed, even if our proposal considers more metrics to be optimized compared to the other two objective functions, it allows to reduce this number of changes which gives better routes stability then as a result less packets loss. On the other hand, regarding the influence of the weights on the combined metrics especially when the energy metric weight is greater than RSSI weight, our proposition shows a decrease in the PDR since that the energy metric is instantaneous which influences more the rank processing then leads to more routing path changes.

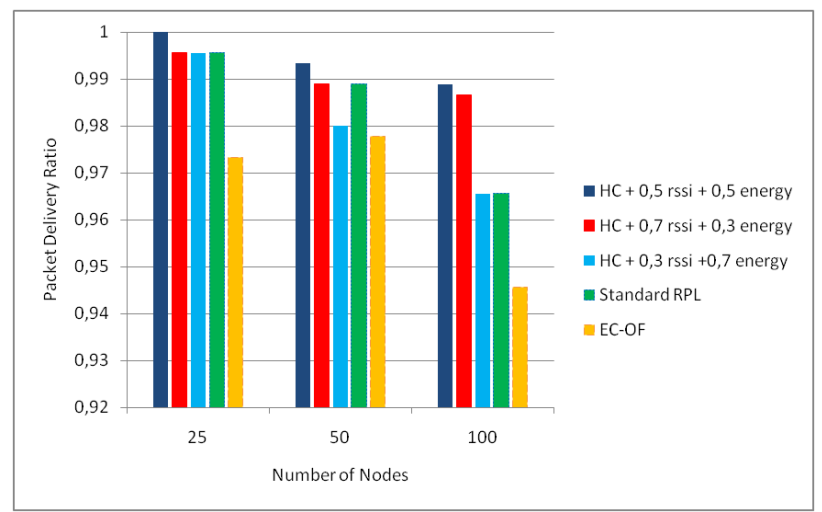

Figure 2. Packet delivery ratio for different network densities 


\subsubsection{Analysis of power consumption}

A glance on Figure 3 shows that HOFESA considerably decrease the power consumption since it is based on combining hop count, energy and RSSI. Indeed, the first metric leads to minimize the hops to the sink which induces technically less energy consumption in retransmission. The second metric tend to offer a path with low power consumption and the third metric gives a good link quality in routing path which is good to avoid the retransmission caused by poor links. On the other hand, as we can see in section 4.1 .5 our proposal induces less DIO control packets which avoids the excess energy consumption in the processing, sending, and reception of these packets. Regarding the influence of the weights on HOFESA, when the superiority is given to the energy metric, there is a slight increase in energy consumption because, as mentioned before, it continually changes which leads to more preferred parents changes.

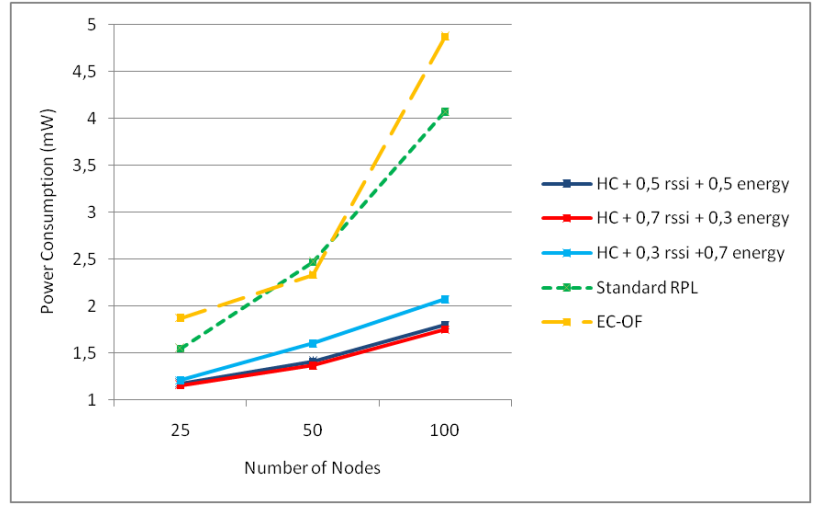

Figure 3. Power consumption for different network densities

\subsubsection{Analysis of churn}

The churn is a essential parameter that several research papers neglect whereas it is very important for evaluating the routing protocol performances. The goal is to minimize it with non-zero values in order to not affect the network stability and optimize the routing paths continously with less energy consumption loss in changing the prefered parent. As noticed in Figure 4, our proposition with a dominant weight on the energy metric tends to frequently change its preferred parents, which leads to a performance deterioration in terms of PDR and energy consumption as we could see previously. However, our proposal with a dominant weight for the RSSI metric reveals an appropriate churn reflecting thus a good PDR with the lower power consumption.

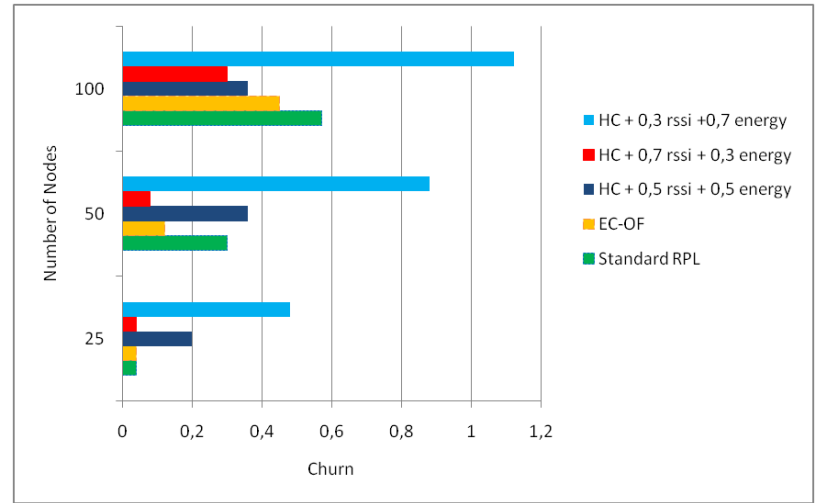

Figure 4. Churn for different network densities

\subsubsection{Analysis of convergence time}

Convergence time reflects one of the properties of real-time performance that a routing protocol can provide. A short convergence time means that the protocol allows the nodes to quickly construct the network arborescence. As can be seen in Figure 5, the convergence time increases as the density is higher. The results 
reveals that HOFESA can converge faster notably when increasing the number of nodes in the network. This can be explained by the fact that each node, in its rank processing, refers to its own metrics without waiting for those of its candidate parent.

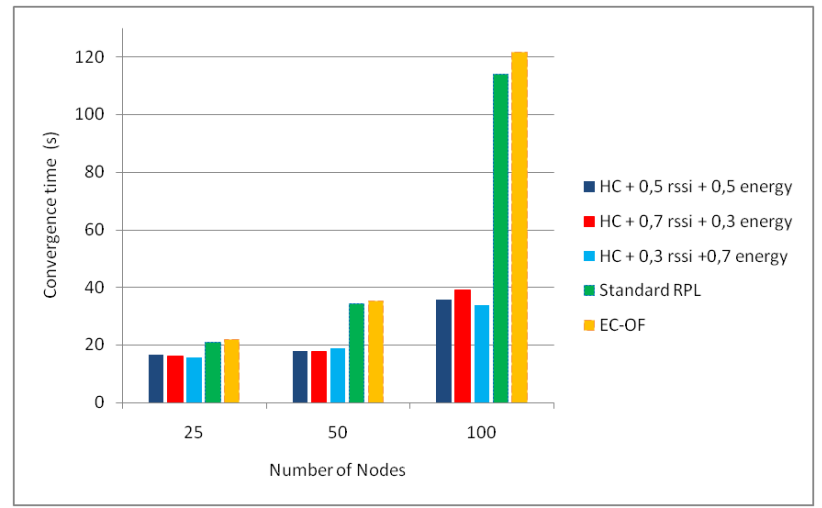

Figure 5. Convergence time for different network densities

\subsubsection{Analysis of DIO control packets}

The aftereffect of network stability is provided by the number of DIO control packets. Indeed, as shown in Figure 6, our proposal can provide a low number of DIO packets compared to Standard-RPL and OFEC due to the optimized paths selected using combined metrics. Concerning the weight's effect, we can notice that the instability induced when the energy metric have the higher weight, the number of DIO automatically increases.

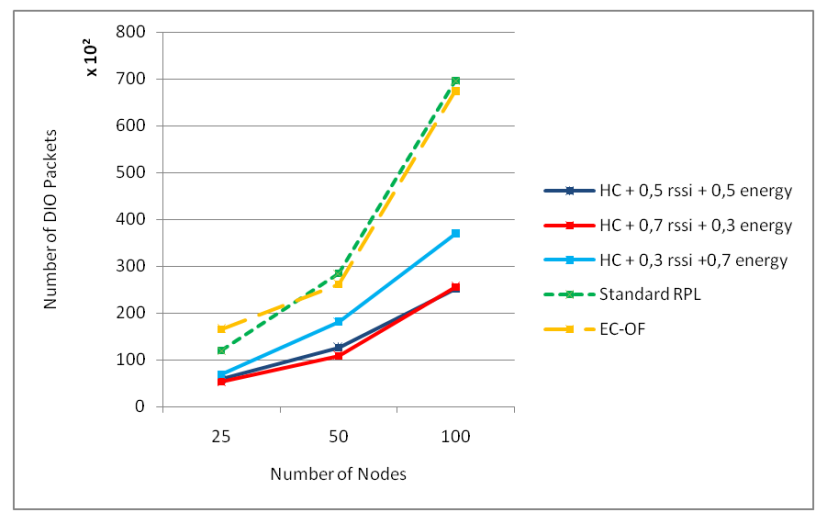

Figure 6. DIO control packets for different network densities

\subsection{Stability effect on HOFESA performances}

This part focuses on the the stability effect induced by empirical threshold defined previously in (8). For this, we were interested in our proposal where the churn is very large, namely $\mathrm{HC}+0.3 \mathrm{rssi}+0.7$ energy. Indeed, we obtained this threshold of stability empirically in order to find the optimal performances. From the Tables 3, 4 and 5, it can be seen that empirical threshold can decrease the churn, the convergence time as well as a provide good PDR with less power consumption and DIO packets.

Table 3. Empirical threshold effect on HOFESA in density of 25 nodes

\begin{tabular}{cccccc}
\hline Stability & PDR & Power(mW) & churn & DIO & Convergence(s) \\
\hline Static threshold & 0,995 & 1,207 & 0,48 & 6864 & 15,769 \\
Empirical threshold & 1 & 1,166 & 0,12 & 5700 & 15,490 \\
\hline
\end{tabular}


Table 4. Empirical threshold effect on HOFESA in density of 50 nodes

\begin{tabular}{cccccc}
\hline Stability & PDR & Power(mW) & churn & DIO & Convergence(s) \\
\hline Static threshold & 0,980 & 1,599 & 0,88 & 18130 & 18,679 \\
Empirical threshold & 0,988 & 1,421 & 0,26 & 12205 & 16,110 \\
\hline
\end{tabular}

Table 5. Empirical threshold effect on HOFESA in density of 100 nodes

\begin{tabular}{cccccc}
\hline Stability & PDR & Power $(\mathrm{mW})$ & churn & DIO & Convergence(s) \\
\hline Static threshold & 0,965 & 2,07 & 1,12 & 36956 & 33,680 \\
Empirical threshold & 0,966 & 2 & 0,66 & 34342 & 27,789 \\
\hline
\end{tabular}

\section{CONCLUSION}

In this paper, an improvement of RPL routing protocol based on its objective function was proposed. Our approach called HOFESA relies on a novel method for rank processing using three metrics namely Hop count, RSSI and Energy consumption to surmount the single routing metric limits. The metrics amalgamation is based on weights that identifies the more influencing in the rank calculation. The network stability is also taken into account by introduced static and empirical threshold to limit the number of parents changes and improve the proposal performances in terms of quality of services. The designed HOFESA, evaluated against StandardRPL and EC-OF, showed an enhancement in packet delivery ratio, lower power consumption, convergence time and DIO control messages as well as it ensure network stability through an adequate churn. In the future work, we will focus on combination more metrics as traffic management metric, ETX, buffer of nodes and delay, to bring out better performances.

\section{ACKNOWLEDGEMENT}

This work has been supported by the Technology of Information and Communication Center of university Hassan II Casablanca as a part of the Big data and Connected objects research project, and the National Center for Scientific and Technical Research in Morocco (CNRST).

\section{REFERENCES}

[1] I. Yaqoob, et al., "Internet of Things architecture: Recent advances, taxonomy, requirements, and open challenges," IEEE Wireless Communications, vol. 24, no. 3, pp. 10-16, 2017.

[2] A. Musaddiq, et al., "A survey on resource management in IoT operating systems," IEEE Access, vol. 6, pp. 8459-8482, 2018.

[3] M. A. Naeem, et al., "A periodic caching strategy solution for the smart city in information-centric Internet of Things," Sustainability, vol. 10, no. 7, pp. 2576, 2018, doi: https://doi.org/10.3390/su10072576.

[4] K. Yoshigoe, et al., "Overcoming invasion of privacy in smart home environment with synthetic packet injection," TRON Symposium (TRONSHOW), 2015.

[5] Y. A. Qadri, et al., "The Future of Healthcare Internet of Things: A Survey of Emerging Technologies," IEEE Communications Surveys and Tutorials, vol. 22, no. 2, pp. 1121-1167, 2020.

[6] R. Ali, et al., "Q-learning-enabled channel access in next-generation dense wireless networks for IoTbased eHealth systems," EURASIP Journal on Wireless Communications and Networking, vol. 2019, pp. 1-12, 2019.

[7] L. D. Xu, Wu He, and Shancang Li "Internet of Things in industries: A survey," IEEE Transactions on Industrial Informatics, vol. 10, no. 4, pp. 2233-2243, 2014.

[8] V. C. Gungor, et al., "Smart grid technologies: Communication technologies and standards," IEEE Transactions on Industrial Informatics, vol. 7, no. 4, pp. 529-539, 2011.

[9] R. Alexander, et al., "RPL: IPv6 Routing Protocol for Low-Power and Lossy Networks. Internet Engineering Task Force(IETF)," RFC 6550, pp. 1-157, 2012.

[10] A. E. Hassani, Aicha Sahel, and Abdelmajid Badri "A New Objective Function Based on Additive Combination of Node and Link Metrics as a Mechanism Path Selection for RPL Protocol," International Journal of Communication Networks and Information Security, vol. 12, no. 1, pp. 63-68, 2020.

[11] O. Gnawali and P. Levis, "The Minimum Rank with Hysteresis Objective Function. Internet Engineering Task Force(IETF)," RFC 6719, 2012. 
[12] P. Thubert, "Objective Function Zero for the Routing Protocol for Low-Power and Lossy Networks (RPL). Internet Engineering Task Force(IETF)," RFC 6552, 2012.

[13] A. E. Hassani, Aïcha Sahel, and Abdelmajid Badri "Impact of RPL Objective Functions on Energy Consumption in Ipv6 based Wireless Sensor Networks," Colloque International sur les Objets et Systèmes Connectés, 2019.

[14] S. A. Hakeem, Shimaa A., Anar A. Hady, and HyungWon Kim, "RPL routing protocol performance in smart grid applications based wireless sensors: Experimental and simulated analysis," Electronics, vol. 8, no. 2, pp. 1-23, 2019.

[15] A. E. Hassani, et al., "Assessment of a proactive routing protocol RPL in Ipv6 based wireless sensor networks," Third International Conference on Intelligent Computing in Data Sciences (ICDS), pp. 1-7, 2019.

[16] W. Xiao, et al., "An optimization of the object function for routing protocol of low-power and Lossy networks," 2nd International Conference on Systems and Informatics, pp. 515-519, 2015.

[17] P. O. Kamgueu, et al., "Energy-based routing metric for RPL," HAL archives, vol. 14, 2013.

[18] P. Sanmartin, et al., "Sigma routing metric for rpl protocol," Sensors, vol. 18, no. 4, pp. 1277, 2018.

[19] P. Gonizzi, Riccardo Monica, and Gianluigi Ferrari "Design and evaluation of a delay-efficient RPL routing metric," 9th International Wireless Communications and Mobile Computing Conference, pp. 15731577,2013

[20] J. Nassar, et al., "Multiple instances QoS routing in RPL: Application to smart grids," Sensors, vol. 18, no. 8, pp. 1-16, 2018.

[21] L. Gao, Zengwei Zheng, and Meimei Huo "Improvement of RPL protocol algorithm for smart grid," International Conference on Communication Technology Proceedings, pp. 927-930, 2019.

[22] S. N. Mishra, et al., "Eha-rpl: A composite routing technique in iot application networks," Advances in Intelligent Systems and Computing, vol. 1045, pp. 645-657, 2020.

[23] H. Al-Kashoash, et al., "Congestion-Aware Routing Protocol for 6LoWPANs," Congestion Control for 6LoWPAN Wireless Sensor Networks: Toward the Internet of Things, pp. 95-107, 2020.

[24] H. Araujo, et al., "A proposal for IoT dynamic routes selection based on contextual information," Sensors, vol. 18, no. 2, pp. 1-16, 2018.

[25] S. Sankar, and P. Srinivasan, "Fuzzy logic based energy aware routing protocol for internet of things," International Journal of Intelligent Systems and Applications, vol. 10, pp. 11-19, 2018.

[26] H. Lamaazi, and N. Benamar, "OF-EC: A novel energy consumption aware objective function for RPL based on fuzzy logic,' Journal of Network and Computer Applications, vol. 117, pp. 42-58, 2018.

[27] H. Lamaazi, and N. Benamar, "RPL enhancement using a new objective function based on combined metrics," 13th International Wireless Communications and Mobile Computing Conference (IWCMC), 2017. 